\title{
Antenatal and perinatal factors associated with new bornbehavior: A prospective cohort study
}

\author{
Effrosine Tsekoura ${ }^{1}$, Kleopatra Chrelia ${ }^{2}$, Abraham Pouliakis ${ }^{3}$, \\ Achilleas Attilakos ${ }^{4}$, Paraskevi Matsota ${ }^{5}$
}

'MD, PhD, Pediatric Department, Asklepieion, General Hospital, Athens, Greece

2Third Department of Pediatrics, "Attikon" Hospital, National and Kapodistrian University of Athens, Greece ${ }^{3}$ Second Department of Pathology, "Attikon" Hospital, National and Kapodistrian University of Athens, Greece ${ }^{4} \mathrm{MD}, \mathrm{PhD}$, Third Department of Pediatrics, "Attikon" Hospital, National and Kapodistrian University of Athens, Greece ${ }^{5} \mathrm{MD}, \mathrm{PhD}$, 2nd Department of Anesthesiology, "Attikon" Hospital, National and Kapodistrian University of Athens, Greece

\section{Corresponding Author}

Chrelia Kleopatra MD, 64 Mesogeion str, Athens 11526 Greece, e-mail: chreliakleopatra@gmail.com

\section{Abstract}

The idea that the newborn is an uncharted and therefore pliable organism, which can be formed according to the will of its parents, has long been rejected, especially after the existence of rules of behavior not only in full-term but also in marginally premature newborns, which are affected by various both prenatal and perinatal factors. The ability of newborns to determine the rules of their behavior according to the stimuli they receive from internal and external factors, has been the subject of many years of research and has led pediatricians and other health professionals to develop a model for grading these newborn abilities. Thus, the Neonatal Behavioral Assessment Scale (NBAS) was created, and it has been used by researchers for more than 40 years. It is a research tool with wide application, as many of the points it addresses, can be integrated into other means of assessing the newborn's abilities. This scale also has an ever-evolving application as a clinical tool in parental assessment.

Key words: Newborn, neonatal, behavior, antenatal, perinatal

\section{Introduction}

The idea that the newborn is an uncharted and therefore pliable organism, which can be formed according to the will of its parents, has long been rejected, especially after the existence of rules of behavior not only in full-term but also in marginally premature newborns, which are affected by various both prenatal and perinatal factors. The ability of newborns to determine the rules of their behavior according to the stimuli they receive from internal and external factors, has been the subject of many 
years of research and has led pediatricians and other health professionals to develop a model for grading these newborn abilities. Thus, the Neonatal Behavioral Assessment Scale (NBAS) was created, and it has been used by researchers for more than 40 years ${ }^{1-3}$. It is a research tool with wide application, as many of the points it addresses, can be integrated into other means of assessing the newborn's abilities. This scale also has an ever-evolving application as a clinical tool in parental assessment.

When a newborn of a high-risk pregnancy is born, then the risk factor, whether it is premature birth, drug exposure, congenital anomaly or even traumatic birth, will affect its ability to maintain a normal and behavioral organization, a result that may be temporary or long-term depending on the cause of the disorganization. Usually, high-risk newborns are easily over-excited and their behavioral traits are difficult to interpret. They have difficulty dealing with environmental stimuli, show normal disorganization such as discoloration, increased respiratory effort, poor temperature regulation and impaired function of the visceral and digestive systems. They often sleep poorly, find it difficult to get used to the stimuli and have problems that cause a relaxed tone and posture. All or any of these signs of disorganization in the newborn affect his ability to interact with his caregiver and to support his efforts for self-regulation.

In the present study we sought to investigate the antenatal and perinatal factors that seem to influence the regulation of term and late preterm offspring.

\section{Materials and methods}

Between August 2014 and January 2016, 150 newborns were examined, however, data were available for inclusion and analysis in the present study in 67 newborns and their mothers. These newborns were examined using i) 28 Behavioral Items that were rated from 9 to 1, ii) 18 neonatal reflexes (Reflex Items) with a score from 0 to 3 , evaluating the neurological behavior of the infant and iii) 7 Supplementary Items with a score from 9 to 1 , where the range and quality of behavior for high-risk newborns is captured so that large abnormalities or asymmetries are identified.

These 53 total fields were evaluated and then graded accordingly. These measurements were made in the 2nd or 3rd day of newborn life. A special questionnaire (35 questions) was also provided that correlated maternal feelings concerning the relation of mother-newborn. The questionnaire was administered and answered by mothers whose infants were examined on the NBAS test during their three-day hospitalization at the maternity hospital.

The data were recorded in a Microsoft Excel file so that each column corresponds to a measured parameter and each row to the data of a newborn / mother. This type of recording facilitates the export of aggregated data for the study of data. The statistical analysis was performed with the software package SAS 9.4 for Windows as well as with the help of Excel 2007 for Windows (Microsoft corporation) and the level of statistical significance was considered as $\mathrm{p}<0.05$. For the analysis of the data expressed in numerical value (eg newborn weight) the analysis was performed using the Kruskal-Wallis method while for the data expressed in a categorical way e.g. newborn sheet, the chi-square test was performed. The significance level was set lower than 0.05 .

Of the 67 newborns examined, 64 were included in the analysis because in three of them the assessment of behavior was done on the first day of birth, while according to the protocol the assessment is performed on the second or third day. A complete record of behavioral data was available for these infants.

More specifically, for each element of behavior that was measured numerically we recorded the number of measurements that were successful $(\mathrm{N})$, the mean (Mean), the standard deviation (Std Dev), the maximum (Maximum) and minimum (Minimum) 
value that were measured as also the value below which 25,50 and $75 \%$ of the measurements are concentrated (25th Pctl, 50th Pctl, 75th Pctl) and the range of the measurement area where $50 \%$ of the average measurements (Quartile Range) are concentrated.

\section{Results}

Overall, 39 boys and 25 girls were examined, of which 25 were born with normal labor, 2 with assisted labor and 37 with cesarean section, all in good general condition, at term or late preterm period (gestational week at delivery ranging betwee 37 and 41 weeks (Table 1).

Sixty-two of these newborns were born with an Apgar Score above 8, with an average value of 9 and 2 newborns with an Apgar Score below 5. The birth weight ranged from $2770 \mathrm{gr}$ to $4300 \mathrm{gr}$ with an average value of $3245 \mathrm{gr}$. The mothers were receiving medication for their pregnancy, but at the same time 11 of them were also treated for thyroid disease.

Perinatal anesthesia was offered in 51 cases. Specifically, 15 women had spinal anesthesia, 17 women epidural, 19 women combined spinal and epidural and 3 women general anesthesia.

In our study, a statistically significant correlation was observed $(\mathrm{p}=0.04)$ between the educational level of the mother and the offspring's regulation (Table 2). Values> 19 indicate hyper-reactivity, while values $<19$ indicate hypo-reactivity. Offspring of mothers with low educational level, had an average value of regulation of 21 . In the middle educational level, the average value of regulation was 19 and in offspring of mothers with high educational level, the average value of regulation was 18 . The optimal value is considered to be $<19$. Children in all 3 groups showed high performance and hyper-reactivity, however children from mothers with high educational level had better outcomes compared to the other two categories. Maternal education was evaluated as elemental when primary education was only completed, middle when high school was completed and high educational when college was completed.

The educational level of the mother seems to influence the behavior of the newborns. Thus, infants of mothers with low educational level usually show a hyper-reactive behavior. External stimuli are improved in children of mothers with a high level of education. We speculate that the hyperreactivity of newborns is related to parturient's hospitalization and the conditions prevailing in the maternity hospital. It is hypothesized that women of low or medium level of education were necessarily in a noisier environment in the maternity hospital, since they are usually hospitalized in crowded environments.

Offspring of mothers who are entertained or not, during pregnancy, show a high score $(>10)$. Watching theatrical or cinematic performances during pregnancy or none of them was considered as entertainment for the mother. Regarding the autonomous system, a statistically significant difference was observed between the groups $(p=0.01)$. In the autonomous system, tremor, shaking, and color instability were evaluated using a limit of 10 . Even though in both groups children scored high on the autonomous system, offspirng of mothers who had no form of entertainment during pregnancy scored higher compared to the autonomous system (15 vs. $13, p=0.01$ ). It is possible that higher performance of newborns of mothers who did not have entertainment during pregnancy, is attributed to cases of stressful causes during daily activities, including work.

Maternal relaxation was assessed by the nature of mother`s family life (quiet family environment or experiencing quarrels or domestic violence). In our study, differences in neonatal behavior observed among these two groups were not significant (Table 3). Recent studies of pregnant women with known depression have found that newborns have behavioral disorders, which are recorded using the 


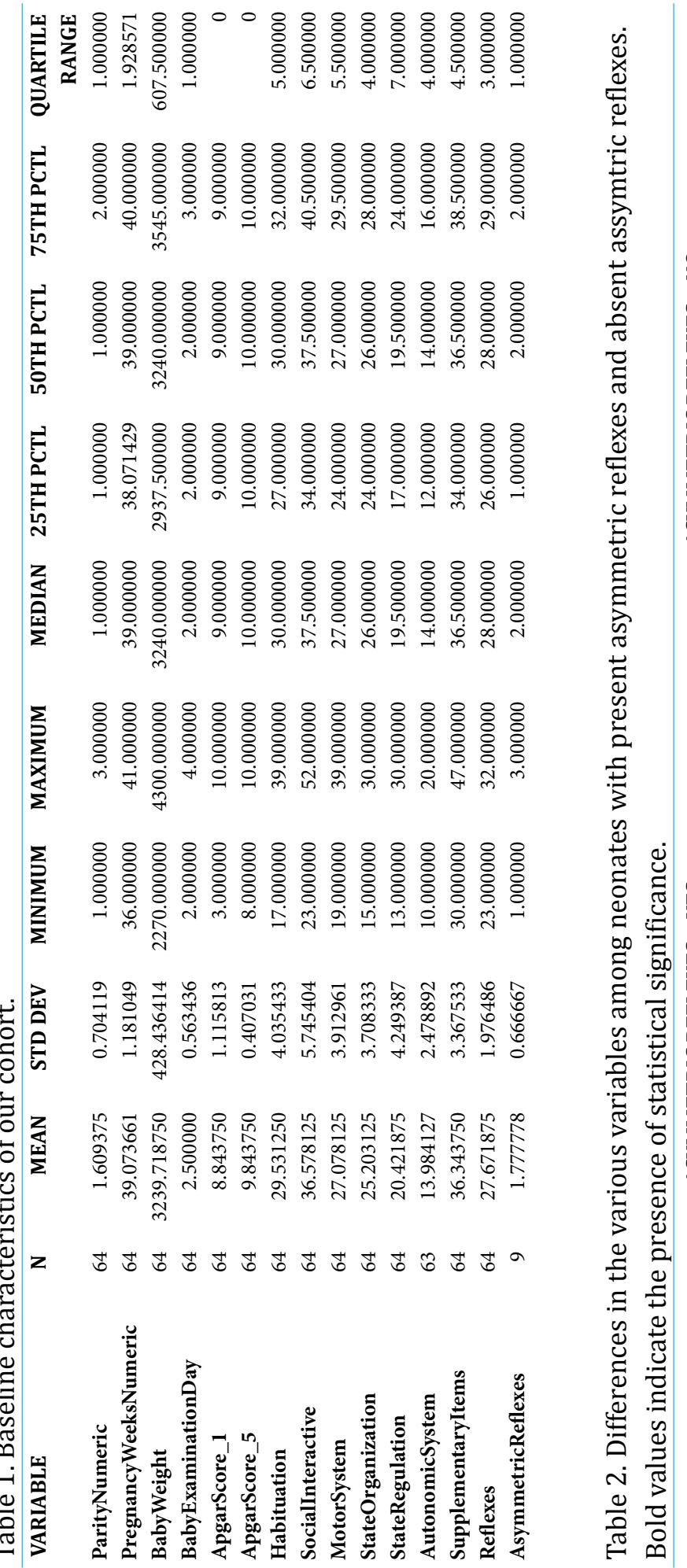

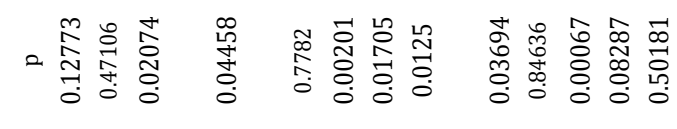

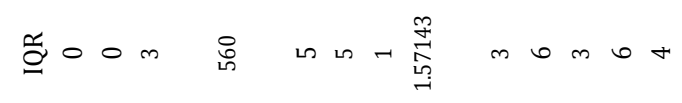

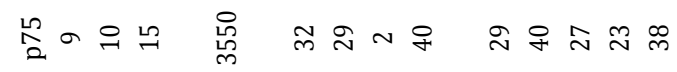

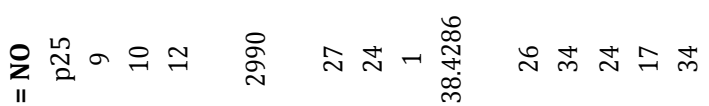

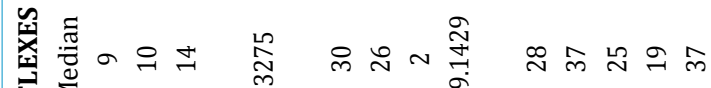

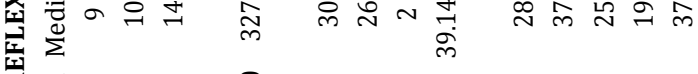

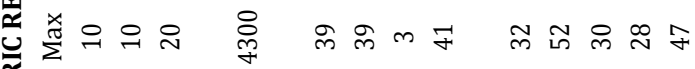

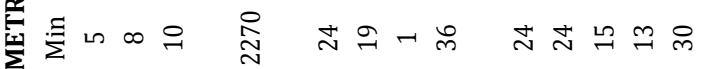

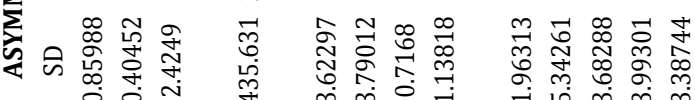
๘

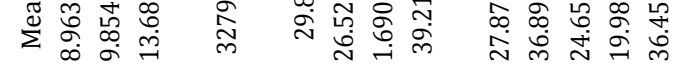

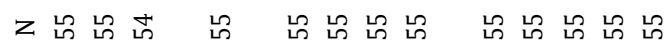

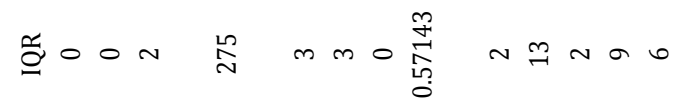

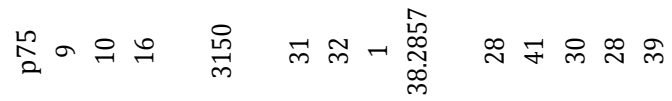

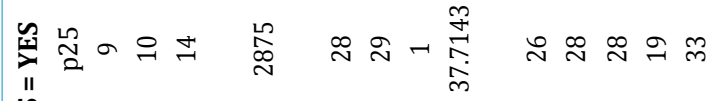

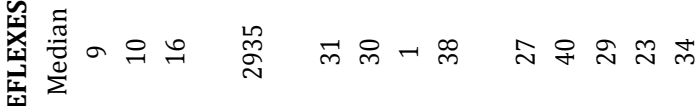

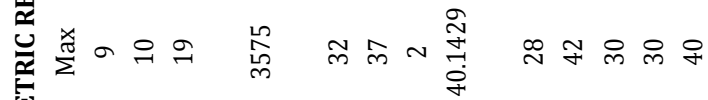

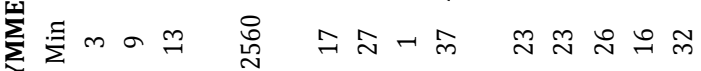

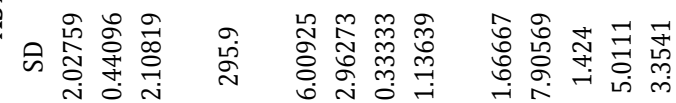

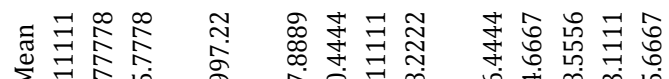

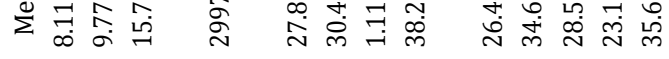
zana a ara aबa 
Table 3. Neonatal characteristics according to the state of calmness of mother.

\begin{tabular}{|c|c|c|c|c|c|c|c|c|c|}
\hline & \multicolumn{4}{|c|}{ CALM STATE } & \multicolumn{4}{|c|}{ NON CALM STATE } & \multirow[b]{2}{*}{$P$-VALUE } \\
\hline & $\mathbf{N}$ & $\begin{array}{l}\text { MEAN } \\
\text { VALUE }\end{array}$ & SD & $\begin{array}{c}\text { MEDIAN } \\
\text { VALUE }\end{array}$ & $\mathbf{N}$ & $\begin{array}{l}\text { MEAN } \\
\text { VALUE }\end{array}$ & SD & $\begin{array}{c}\text { MEDIAN } \\
\text { VALUE }\end{array}$ & \\
\hline Asymmetric reflexes & 8 & 1,75 & 0,70711 & 2 & 1 & 2 & . & 2 & 0,66501 \\
\hline Autonomic system & 48 & 13,9167 & 2,54185 & 14 & 15 & 14,2 & 2,33605 & 14 & 0,51414 \\
\hline Habitutation & 49 & 29,551 & 4,04692 & 30 & 15 & 29,4667 & 4,13809 & 29 & 0,49807 \\
\hline Motor system & 49 & 27,1429 & 3,66856 & 27 & 15 & 26,8667 & 4,76395 & 26 & 0,62119 \\
\hline Reflexes & 49 & 27,6327 & 2,12812 & 28 & 15 & 27,8 & 1,42428 & 28 & 0,75389 \\
\hline Social Interaction & 49 & 36,9388 & 6,10126 & 38 & 15 & 35,4 & 4,35562 & 37 & 0,1869 \\
\hline State organization & 49 & 25,2041 & 3,8567 & 26 & 15 & 25,2 & 3,29935 & 25 & 0,72567 \\
\hline State regulation & 49 & 20,5306 & 4,083 & 20 & 15 & 20,0667 & 4,8912 & 19 & 0,567 \\
\hline Supplementary Items & 49 & 36,6122 & 3,5108 & 37 & 15 & 35,4667 & 2,77403 & 36 & 0,23711 \\
\hline
\end{tabular}

NBAS scale. According to the authors, these changes in neonatal behavior are attributed to effects of inutero programming, epigenetic factors or genetic predisposition.

In our study we evaluated how the use of mobile phones with the help of headphones or handsfree or none of thoseaffected the behavioral parameters of newborns. The duration of the use was not recorded. A statistically significant difference was observed in the autonomous system ( $p=0.08)$. It therefore seems that the newborns of mothers who did not use a mobile phone had worse regulation. No differences were observed with respect to other confounding factors. We suspect that the newborns of mothers who talked on their cell phones performed better in the autonomous system, due to the fact that their mothers were in a calmer state during pregnancy, that was attributed to the use of their mobile phones.

In the autonomous system, differences were also observed in relation to the use or not of a microwave oven. Children of mothers who did not use a microwave oven showed better regulation in the autonomous system ( 14.75 vs $13.51, p=0.04$ ). This may be due to the fact that mothers who used a microwave oven, lived a fast and intense life, which resulted in a corresponding effect on the behavior of newborns.

Newborns of mothers who used a computer or not were also studied comparatively. The duration of use was not recorded. A negative answer was considered as zero use. No statistically significant differences were observed in the behavior of newborns regarding the use of computers by mothers.

There was a statistically significant difference in the use of magnesium in terms of maternal sociability. Newborns of mothers taking magnesium had a high reactivity (regulatory value 40 with a limit of 35 ). The high reactivity of these newborns is attributed to the poorsocial life of mothers, which might be the result of premature contractions and muscle contractions (cramps) for which they received magnesium preparations. A relatively recent meta-analysis of the Cochrane Database states that there are not enough high-quality studies to document the benefit of taking magnesium supplements during pregnancy in infants ${ }^{4}$.

Regarding the behavior of newborn mothers who received or did not receive progesterone during pregnancy, no statistically significant differences were observed in our study. 
In cases where mothers received thyrohormone, there were differences in the behavior of the newborns. Offspring of mothers receiving thyrohormone had more intense reflexes than children whose mothers did not receive thyrohormone (27.55 vs 28.64 $\mathrm{p}=0.04)$. In the literature, a positive correlation is documented between the levels of thyroid hormones in the newborn's blood, with the quality and intensity of specific reflexes (Tan, 1994). Therefore, in neonates of mothers receiving exogenous thyroxine, an increase in thyroxine in the peripheral blood is expected, and consequently an effect on the reflexes.

No differences in the behavioral parameters of the newborns were observed between first-born and multi-born mothers.

Concerning $1 `$ Apgar Score values no differences were observed in the additional data. The additional data that were evaluated were: the quality of vigilance, the ability to pay attention, the ease of the examination, the irritability, the intensity, the duration of general application, and the general context of the examiner (objectivity). Children with low Apgar Score during the 1 minute a life and a score of complementary components below 9 were more irritable than children with an Apgar Score above 9 ( 38 vs $35, \mathrm{p}=0.02$ ).

No differences were observed between infants regarding the Apgar Score at 5 : This is probably attributed to the adaptation of newborns to the external environment by the 5th minute of life.

The birth weight of newborns significantly affected the motor system of offspring (generalized tone, maturity of the motor sector, pulling in a sitting position and defensive movements). Newborns with a birth weight over $3 \mathrm{kgr}$, seem to have difficulty in organization, compared to newborns with a birth weight below $3 \mathrm{Kgr}$ (average value 24.42 versus 27.42 respectively, $p=0.001$ ). A value below 24 in the organization indicates hyperreactivity (more difficult organization). Also, neonates with high birth weight seem to have a lower score in the locomotor system compared to low birth weight neonates (26.10 vs. 29.16 respectively, $\mathrm{p}=0.002$ ). Recent studies support the existence of differences in motor behavior in preterm infants of low birth weight. It is reported that in these cases, one of the prognostic indicators of the future neurodevelopmental development of the newborn is the birth weight. Therefore, birth weight is, in our study, an indicator of neurodevelopmental development, as expressed by the NBAS scale.

In the comparison of newborn mothers who may or may not have had thyroid disease (during pregnancy or pre-existing), significant differences of reflexes were observed. Specifically,neonates of mothers with thyroid disease had values within normal limits, compared to the neonates of mothers who did not have thyroid disease ( 28 vs. $27, \mathrm{p}$ $=0.02$ ). It is believe that the levels of the thyroid hormone of the newborn are probably affected in womewho receiveexogenous thyroxine, as well as among mothers with thyroid disease, which in the literature seems to affect the expression of specific reflexes.

Regarding diabetes, differences are observed in the motor system, with the newborns of diabetic mothers having better reactivity than newborns of mothers without diabetes ( 26 vs. $29, \mathrm{p}=0.002$ ). Pregnant women without diabetes obviously had no reason to pay attention to their diet. It may also be attributed to the fact that the newborns of diabetic mothers are large, more sluggish and therefore quieter motor. There are also statistically significant differences in the field of organization, as newborns of non-diabetic mothers exhibited much better organizational skills (27.42 vs. $24.42 p=0.001$ ). Therefore, newborns born to non-diabetic mothers show better organization but a worse motor system. The literature reports cases of the birth of macrosomic neonates, which show a weaker motor system, without mentioning the presence or absence of diabetes in their moth- 
ers ${ }^{5}$. Other studies report that infants of mothers with pre-existing diabetes mellitus or gestational diabetes mellitus have lower performance on gross motor skills ${ }^{6-8}$.

No differences were observed among offspring of mother that smoked during pregnancy and those that did not. An interesting finding in our study is the fact that the newborns of both mothers who smoked during pregnancy and mothers who did not smoke had similar performance on their neurodevelopmental development. Of course, in relevant literature reports, there are different results between newborn pregnant women who smoked than those who did not smoke, in terms of their neurodevelopmental development ${ }^{9-10}$.

Differences in offspring reflexes and locomotor system were observed among children that breastfed and those that did not. Specifically, offspring that used formula milk had calmer reflexes, while the children with breast milk and the mixed diet had more intense reflexes ( 30 and 29 respectively, $p=0.03$ ). Children who consumed breast milk and a mixed diet also showed better reflex behavior. There are reports that breastfed infants have better neurodevelopmental development, possibly due to the presence of high levels of elements such as vitamin B6 ${ }^{11-12}$.

\section{Conclusion}

The goal of the Brazelton Neonatal Behavioral Assessment Scale (NBAS) is to compile a comprehensive profile of neonatal function describing the full range of neonatal behavior, including abilities and capabilities, and possibilities. This instrument has been shown to be of great clinical value in detecting infants at risk for later developmental problems.The first three years of a child's life are a key period of physical, physiological, cognitive and social development and the caregiver-infant relationship in early infancy plays an important role in influencing these elements of development. A parent's ability to un- derstand their baby's behavior as a communication is a key part of this process. Early, brief interventions such as the NBAS Scale or the Newborn Behavioral Observation (NBO) system are potential methods of improving outcomes for both the infant and the caregiver.

In our study we observed that several antenatal and perinatal factors may affect the newborn`s behavioral assessment score; however, validation of our findings is needed in larger cohorts to ascertain the certainty of our findings.

\section{References}

1. Barbosa M, Moreira J, Tronick E, et al. Neonatal Behavioral Assessment Scale (NBAS): Confirmatory factor analysis of the six behavioral clusters. Early Hum Dev. 2018 Sep; 124: 1-6.

2. Brazelton TB. (1984) Neonatal Behavioral Assessment Scale. 2nd Edn. Clinics in Developmental Medicine No. 88. London: Spastics International Medical Publications.

3. Brazelton TB. Psychophysiologic reactions in the neonate: I. The value of observation of the neonate. The Journal of Pediatrics. Volume 58, Issue 4, April 1961: 508-512.

4. Makrides M, Crosby DD, Shepherd E, Crowther CA. Magnesium supplementation in pregnancy. Cochrane Database of Systematic Reviews 2014, Issue 4. Art. No.: CD000937.

5. Pressler JL, Hepworth JT. Behavior of macrosomic and appropriate-for-gestational-age newborns. J ObstetGynecol Neonatal Nurs. Mar-Apr 1997; 26(2): 198-205.

6. Ornoy A. Growth and neurodevelopmental outcome of children born to mothers with pregestational and gestational diabetes. Pediatr Endocrinol Rev. 2005 Dec;3(2):104-13.

7. Stomnaroska O, Dukovska V, Danilovski D. Neuro Developmental Consequences of Neonatal Hypoglycemia. Pril (MakedonAkadNaukUmet Odd Med 
Nauki). 2020 Sep 1; 41(2): 89-93.

8. Lackovic M, Milicic B, Mihajlovic S et al. Gestational Diabetes and Risk Assessment of Adverse Perinatal Outcomes and Newborns Early Motoric Development. Medicina (Kaunas). 2021 Jul 22; 57(8): 741.

9. Perez-Pereira M, Fernandez P, Gómez-Taibo M, et al. Neurobehavioral development of preterm and full term children: biomedical and environmental influences. Early Hum Dev. 2013 Jun; 89(6): 401-9.

10. Hernández-Martínez C, Arija Val V, Escribano Subías J, Canals Sans J. A longitudinal study on the effects of maternal smoking and secondhand smoke exposure during pregnancy on neonatal neurobe- havior. Early Hum Dev. 2012 Jun; 88(6): 403-8.

11. Ooylan LM, Hart S, Porter KB, Driskell JA. Vitamin B- 6 content of breast milk and neonatal behavioral functioning. J Am Diet Assoc. 2002 Oct; 102(10): 1433-8.

12. Ornoy A. Growth and neurodevelopmental outcome of children born to mothers with pregestational and gestational diabetes. Pediatr Endocrinol Rev. 2005 Dec;3(2):104-13.

Received 07-09-21

Revised 15-09-21

Accepted 21-09-21 